\title{
Fuzzy Rule Based Quality of Service Provisioning in Cognitive Radio Network
}

\author{
NISAR A. LALA ${ }^{1 *}$, G. M. MIR ${ }^{1}$, \\ ALTAF A. BALKHI ${ }^{1}$ and N. A. SOFI ${ }^{2}$
}

${ }^{1}$ College of Agricultural Engineering and Technology, Sher-e-Kashmir University of Agricultural

Sciences \& Technology of Kashmir Srinagar, J\&K, India.

${ }^{2}$ Sher-e-Kashmir University of Agricultural Sciences \& Technology of Kashmir Srinagar, J\&K, India.

\begin{abstract}
Cognitive radio (CR) is a novel technology to resolve the issue of underutilization of wireless spectrum. Quality of service (QoS) provisioning in CR networks to large number of traffic as per their need is not an easy task since no wireless spectrum is available on permanent basis for its operation. In this paper, few critical QoS parameters namely dynamic-availability-ofidle-channels (avail-idle-channel), expected-holding-time-of-idle-channel (HT-idle-channel) and user-mobility are chosen to analyze their impact over quality of service of the communicating cognitive users using rule-based fuzzy decision-making system. The results indicate the relationship of chosen parameters over the QoS of the communicating cognitive users.
\end{abstract}

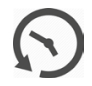

Article History

Received: 19 February 2018

Accepted:19 March 2018

\section{Keywords}

Cognitive radio, Quality of service (QoS) Fuzzy logic.

\section{Introduction}

The spectrum is a range of electromagnetic radiations that enables wireless communication and is managed by government. Day by day, the number of new wireless users and applications are increasing at a very rapid pace and particularly last decade has seen tremendous growth of both. Wireless users worldwide have reached 3.2 billion and were predicted to increase by 100 folds by $2013^{1}$. New applications demand additional allocation of spectrum which is not available and has created artificial scarcity of new allocation of spectrum. The first utilization survey reported that the allocated spectrum remained idle most of the time ${ }^{2}$ and is also function of geographical location and time.

The critical functions of cognitive radio are as under:

\section{Spectrum Sensing}

Spectrum sensing is the important function of the CR. CR uses the function of spectrum sensing of radio environment to detect the spectrum channels not in use at that time known as spectrum hole and use those idle channels for its transmission. The sensing operation should also have the ability to

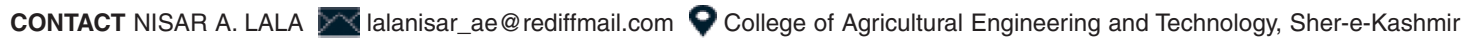
University of Agricultural, Sciences \& Technology of Kashmir Srinagar, J\&K, India.

(C) 2018 The Author(s). Published by Oriental Scientific Publishing Company

This is an 6 Open Access article licensed under a Creative Commons Attribution-NonCommercial-ShareAlike 4.0 International License (https://creativecommons.org/licenses/by-nc-sa/4.0/), which permits unrestricted NonCommercial use, distribution, and reproduction in any medium, provided the original work is properly cited.

To link to this article: http://dx.doi.org/10.13005/ojcst11.01.10 
detect the presence of the PU accurately when the later starts its communication again ${ }^{3}$. The sensing duration is a critical parameter $r^{4,5}$ and depends upon the wireless channel characteristics ${ }^{6,7,8}$.

\section{Spectrum Management}

Spectrum management helps in acquiring the best idle hole for the transmission of CR among large number of available holes found through the operation of sensing. Based on the availability of the spectrum, the suitable hole is allocated to the CR.

\section{Spectrum Mobility}

Spectrum mobility is referred as changing the frequency band during data transmission due to presence of PU on that band. The CR has to switch to another idle hole which is not used by the PU at that time in order to continue its transmission ${ }^{9}$. As soon as the PU needs the current frequency band, the CR has to terminate its transmission and free the presently occupied hole for the PU functioning ${ }^{10}$.

\section{Spectrum Sharing}

Spectrum sharing is an important functionality of CR as it coordinates the traffic between CRs and PUs. It is a challenging task as it requires high degree of cooperation, understanding and coordination between the two.

Over the last decade, the development of wireless communication technology has shown exponential increase and wireless services have evolved from traditional voice service to a wide range of multimedia services. The bandwidth requirement varies from application to application. Hence, providing QoS to these applications as per their need of bandwidth is a critical and challenging task. QoS is the performance level of a service offered by the network to the user in order to achieve deterministic behavior by proper utilization of the network resources. The meaning of QoS changes as per the requirements of the application field. ISO 9000 defined QoS as "the degree to which a set of inherent characteristics fulfils requirements". ITU-T'11,12 defined QoS as "the collective effect of service performance which determines the degree of satisfaction of a user of the service". IETF ${ }^{13}$ considers QoS as "the ability to segment traffic or differentiate between traffic types in order to enable the network to treat certain traffic flows differently from others". The authors of ${ }^{14}$ identified three types of QoS such as Intrinsic QoS, Perceived QoS and Assessed QoS. Intrinsic QoS depends on the performance of the network and is described in terms of objective parameters such as delay, jitter, loss etc. Perceived QoS is the quality perceived by the users and is measured by their average opinion. Assessed QoS refers to the willingness of the users to continue the use of the specific service. In this paper, we have introduced a new QoS parameter known as expected- holding-time-of-idle-channel (HT-idle-channel). HT-idle-channel is the average idle time period of the channel that can be utilized by $\mathrm{CR}$ for its communication before primary user needs that for its transmission. If HT-idle-channel is large, that indicates considerable amount of time is available for CR's communication before necessity of handoff. It is assumed that HT-idle-channeldata is available either provided by spectrum server ${ }^{15}$ or computed by CR itself. Application of fuzzy logic in communication networks offers effective solution when the available information is vague and imprecise. Fuzzy logic found some applications in CR network for transmitting power control ${ }^{16}$ and for optimization ${ }^{17}$. It was applied in CR for controlling the spectrum access ${ }^{18,19}$ and spectrum handoff ${ }^{20,21,22}$. The authors of ${ }^{23,24,25}$ have studied the impact of some of critical parameters over QoS in CR.

\section{Materials and Methods}

In this paper, fuzzy rule-based algorithm is presented that analyzes the impact of three critical parameters namely availability-of-idle-channels (avail-idlechannel), expected-holding-time-of-idle- channel (HT-idle-channel) and user-mobility over the QoS in cognitive radio. We have used Hong and Rappaport traffic model ${ }^{26,27}$ for user-mobility. The model is based on following assumptions:

When a call is initiated, it is assumed that users are uniformly distributed within the cell.

Before initiation of the call, users can move in any direction but after call is initiated users only move in one direction.

The arrival rate of handoff calls is given ${ }^{26,27}$ as

$\lambda H=\left(P_{h}\left(1-B_{o}\right) /\left(1-P_{h h}\left(1-P_{f}^{\prime}\right) \lambda_{o}\right.\right.$ 
$P_{h}$ : gives probability of at least one morehandoff is required without call is being blocked.

$P_{h n}$ : gives probability of the requirement of one more handoff.

$\mathrm{B}_{\mathrm{o}}$ : gives probability of new calls being blocked.

$P_{f}^{\prime}$ : gives probability of unsuccessful handoff.

$\lambda \mathrm{o}:$ gives the arrival rate new calls in a cell.

Fuzzy decision-making system has three inputs

- $\quad$ input 1: (avail-idle-channel) $(\mathrm{KHz})$

- $\quad$ input 2: (HT-idle-channel)(Seconds)

- $\quad$ input 3: user-mobility ( $\mathrm{km} /$ hour)

and the output is the QoS of the communicating users in cognitive radio. Input 1 and input 2 are defined by fuzzy sub-sets represented by low, medium and large, as shown in (2) bounded between $[0,1]$ as shown in Fig. 1(a) fuzzy-set (avail-idle-channel) $=$ fuzzy-set (HT-idlechannel $)=\{$ low, medium, large $\}$

Input 3 is defined by fuzzy sub-sets represented by low, medium and high, as shown in (3) bounded between $[0,1]$ as shown in Fig. 1(b)

fuzzy-set(user-mobility) $=\{$ low, medium, high $\}$

and QoS is defined by fuzzy sub-sets represented by poor, average, good, very-good and excellent, as shown in(4) bounded between $[0,1]$ as shown in Fig. 1(c)

fuzzy-set $($ QoS $)=$ ppoor, average, good, very-good, excellent\}

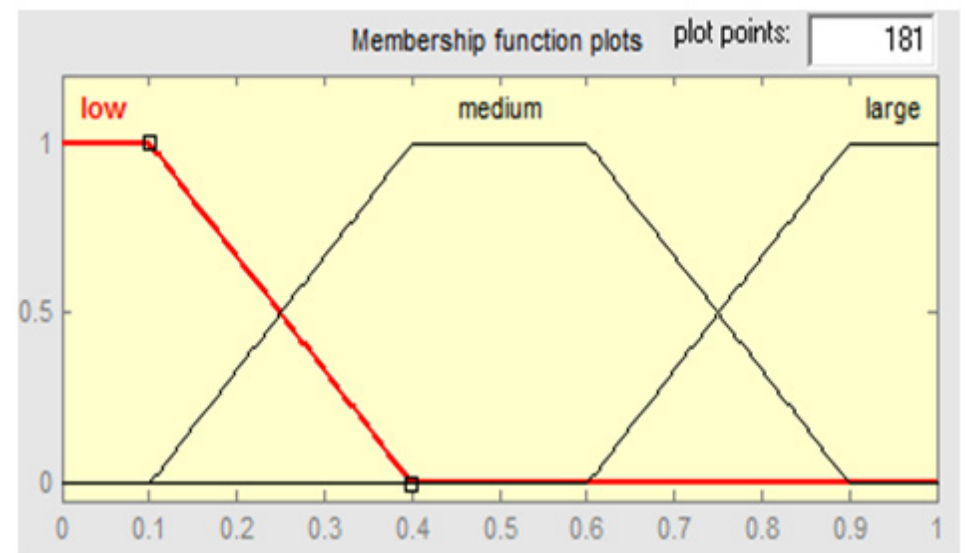

Fig. 1(a): Membership functions for avail-idle-channel \& HT-idle-channel

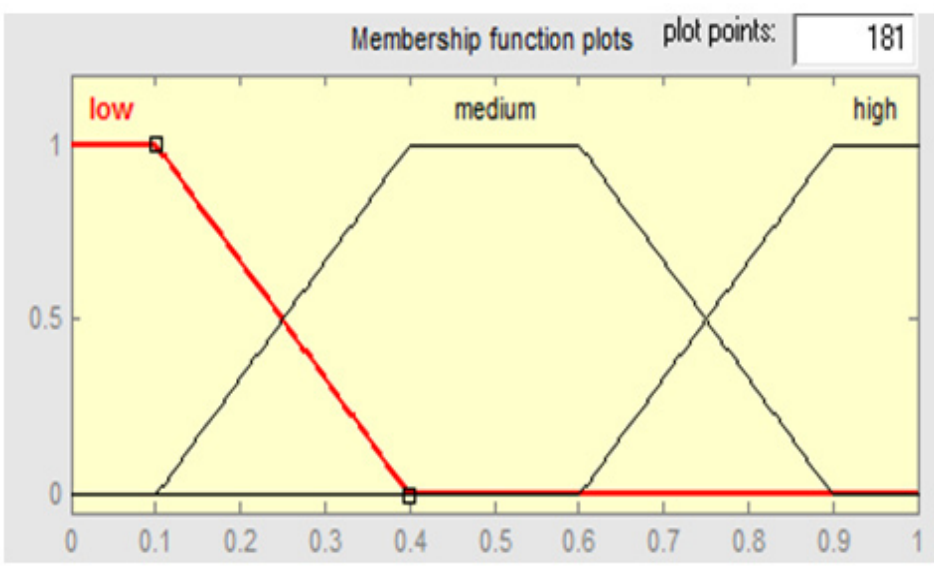

Fig. 1(b): Membership functions for user-mobility 


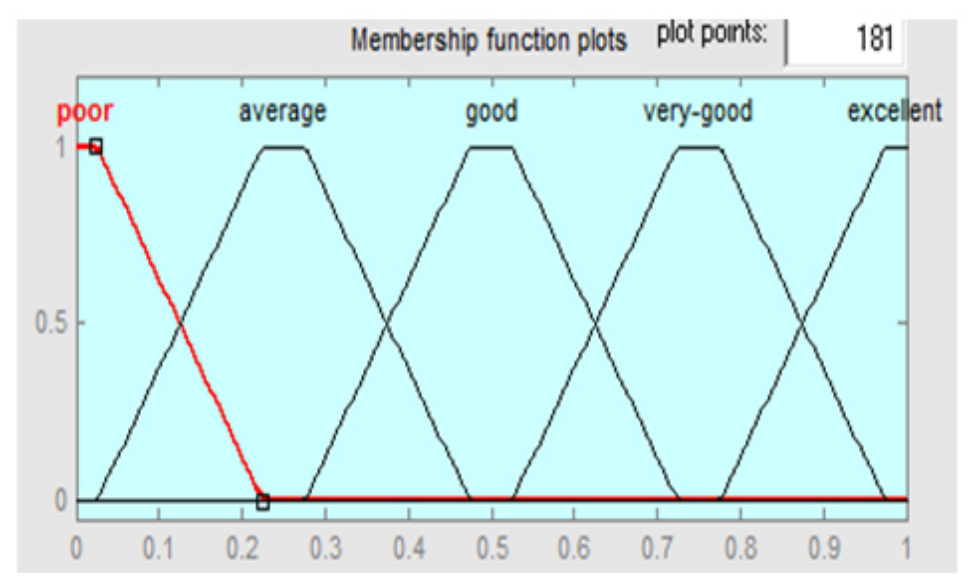

Fig. 1(c): Membership functions for QoS

The proposed algorithm in this paper is analyzed using trapezoidal membership functions for inputs and the output defined by bounded values between $[0,1]$. Fuzzy decision-making system contains 27 rules in its rule-base shown by Table 1 . The fuzzy rules are so designed to combine different combinations of the three contradictory inputs in order to predict the QoS level of the communicating channels. For instance, rule 1 demonstrates that if avail-idle-channel is large, HT-idle-channel is large and user-mobility is low, then the quality of the communicating channel is excellent. This is translated into the scenario in which large number of idle spectrum channels is found through spectrum sensing operation, these idle channels can be used for transmission of $\mathrm{CR}$ for long time without the necessity of handoff and the user is walking slowly or is at stationary position. The QoS of the communication channel is very good that easily satisfies QoS of the communicating cognitive users. Rule 27 demonstrates that if avail-idle-channel is low, HT-idle-channel is low and user-mobility is high, then the quality of the communicating channel is poor. This is translated into the scenario in which small number of idle spectrum channels is available for communication of cognitive users, the available small number of idle channels can be used only for short period of time due to reappearance of PU that forces handoff and the user is moving with very high speed $\geq 100 \mathrm{Kms} / \mathrm{Hr}$. As a result, the QoS of the communication channel is not good and does not satisfy the QoS of the communicating cognitive users. Also rule 18 demonstrates that if avail-idlechannel is medium, HT-idle-channel is low and usermobility is high, then the QoS of the communicating channel is average. This is translated into scenario in which sufficient number of idle spectrum channels are available for communication of cognitive users, the available number of idle channels can only be used for short period of time due to reappearance of $\mathrm{PU}$ that forces handoff and the user is moving with very high speed $>100 \mathrm{Kms} / \mathrm{Hr}$. The QoS of the communicating cognitive users is at threshold level and is just sufficient to satisfy QoS need of the cognitive users. The decision of fuzzy decisionmaking system for input values such as avail-idlechannel $=0.5$ (mid-value), HT-idle-channel $=0.5$ (mid-value), user-mobility $=0.5$ (mid-value) is 0.5 as shown in Fig. 2.

Table 1: Rule-base of fuzzy decision-making system

\begin{tabular}{lllll}
\hline Rule \# & $\begin{array}{l}\text { avail_idle__ } \\
\text { channel }\end{array}$ & $\begin{array}{l}\text { HT_idle_- } \\
\text { channel }\end{array}$ & $\begin{array}{l}\text { user- } \\
\text { mobility }\end{array}$ & QoS \\
\hline 1 & large & large & low & excellent \\
2 & large & large & medium & excellent \\
3 & large & large & high & good \\
4 & large & medium & low & excellent
\end{tabular}


LALA et al., Orient. J. Comp. Sci. \& Technol., Vol. 11(1) 55-63 (2018)

\begin{tabular}{lllll}
5 & large & medium & medium & very-good \\
6 & large & medium & high & good \\
7 & large & low & low & very-good \\
8 & large & low & medium & good \\
9 & large & low & high & average \\
10 & medium & large & low & excellent \\
11 & medium & large & medium & very-good \\
12 & medium & large & high & good \\
13 & medium & medium & low & very-good \\
14 & medium & medium & medium & good \\
15 & medium & medium & high & average \\
16 & medium & low & low & good \\
17 & medium & low & medium & average \\
18 & medium & low & high & average \\
19 & low & large & low & very-good \\
20 & low & large & medium & average \\
21 & low & large & high & average \\
22 & low & medium & low & good \\
23 & low & medium & medium & poor \\
24 & low & medium & high & poor \\
25 & low & low & low & poor \\
26 & low & low & medium & poor \\
27 & low & low & high & poor \\
\hline
\end{tabular}

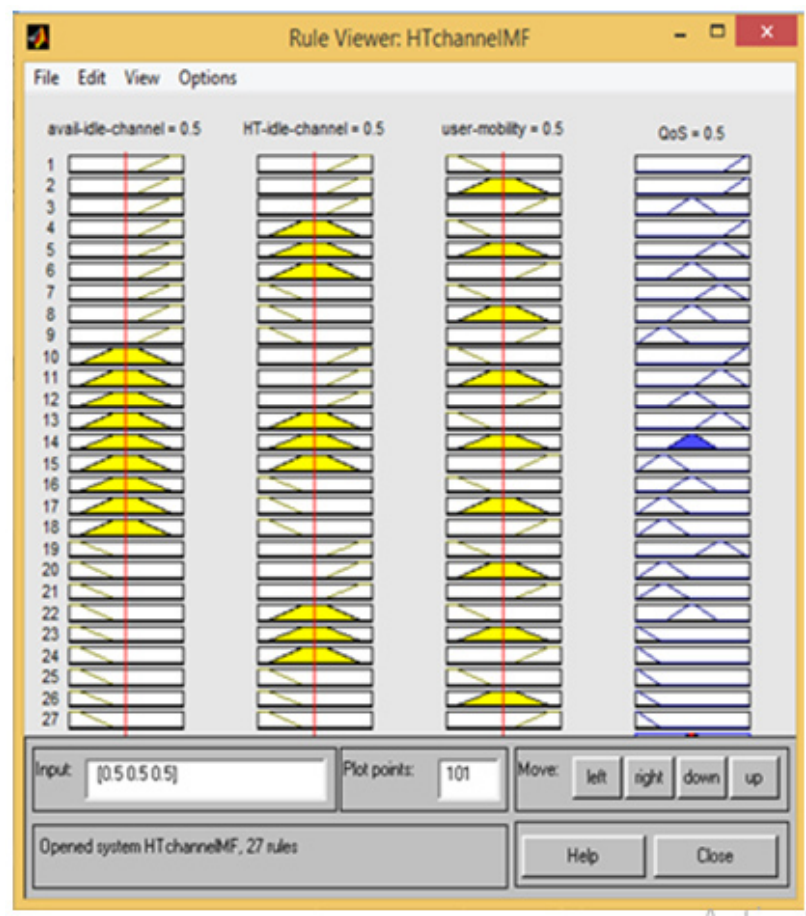

Fig. 2: Decision of fuzzy decision-making system 


\section{Results and Discussions}

The proposed rule-based fuzzy algorithm is analyzed in fuzzy-logic toolbox for static data transfers. The fuzzy decision-making system analyzes the dependence of avail-idle-channel, HT-idle-channel and user-mobility over the QoS of the communicating cognitive users as revealed by Fig.3, Fig.4 and Fig.5. During analysis, the other two parameters are kept fixed at their mid-values (i.e. $=0.5$ ). Fig. 3 revealed that as the number of avail-idle-channel increases i.e idle channels, the QoS of communicating cognitive users increases at fixed mid-values of HT-idle-channel and user-mobility during analysis. Fig. 4 revealed that as the HT-idle-channel increases i.e. usage time of the channel to be used for communication of cognitive users increase, the QoS of communicating cognitive users increase at fixed mid values of availidle-channel and user-mobility during analysis. Fig. 5 revealed that as user-mobility increases i.e. the speed of the user goes on increasing, the QoS of communicating cognitive users decrease at fixed mid-values of HT-idle-channel and avail-idle-channel during analysis.

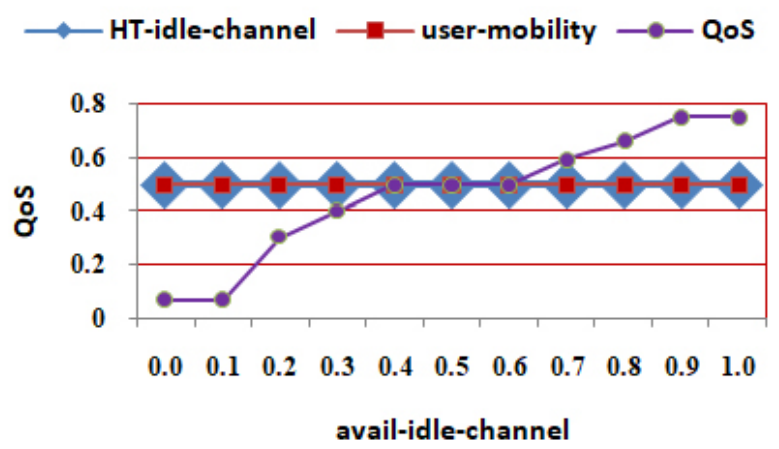

Fig. 3: Impact analysis of avail-idle-channel over QoS
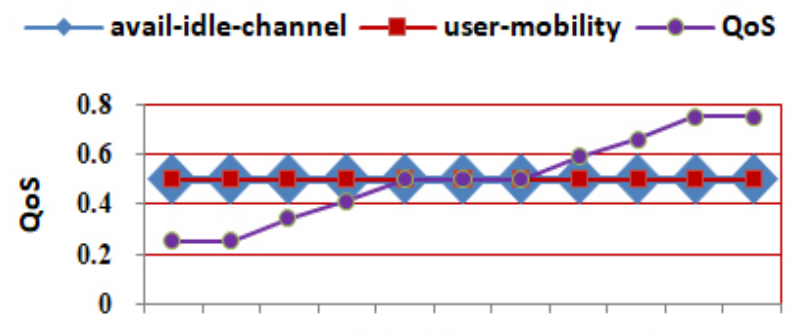

$\begin{array}{lllllllllll}0.0 & 0.1 & 0.2 & 0.3 & 0.4 & 0.5 & 0.6 & 0.7 & 0.8 & 0.9 & 1.0\end{array}$

HT-idle-channel

Fig. 4: Impact analysis of HT-idle-channel over QoS
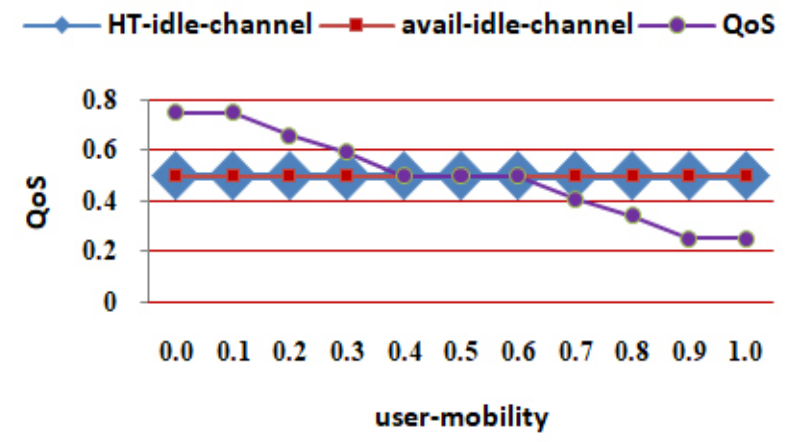

Fig. 5: Impact analysis of user-mobility over QoS 
Fig. 6, Fig. 7 and Fig. 8 reveal the simultaneous dependence of HT-idle-channel and avail-idlechannel, user-mobility and avail-idle-channel, and user-mobility and HT-idle-channel over the QoS of the communicating cognitive users. During analysis, the other parameter is kept fixed at mid-value (i.e. $=0.5$ ). The analytical results revealed that as the avail-idle-channel and HT-idle-channel increases, the QoS of the communicating cognitive users also increases at fixed mid-value of user speed as shown by Fig. 6 and the maximum value of QoS is attained among communicating cognitive users when the HT-idle-channel and avail-idle-channel are at their largest values. Fig. 7 reveals that as the avail-idlechannel increases and speed of the cognitive user decreases, the QoS of the communicating cognitive users increases at fixed mid-value of HT-idle-channel and the maximum value of QoS is attained among communicating cognitive users when the avail-idlechannel is at its largest value and the user remains stationary or moves with very low speed. Fig. 8 reveals that as the HT-idle-channel increases and the speed of the cognitive user decreases, the QoS of the communicating cognitive users increase at fixed mid-value of avail-idle-channel and the maximum value of QoS is attained among communicating cognitive users when the HT-idle-channel is at largest value and the user remains stationary or moves with very low speed.

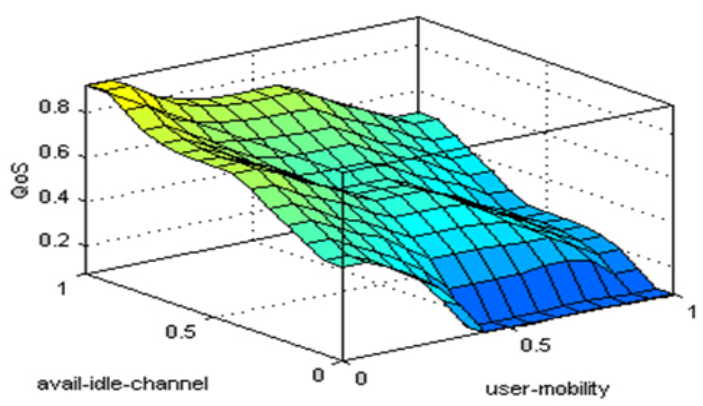

Fig. 7 : Impact analysis of user-mobility and avail-idle-channel over QoS

Fig. 6 : Impact analysis of HT-idle-channel and avail-idle-channel over QoS

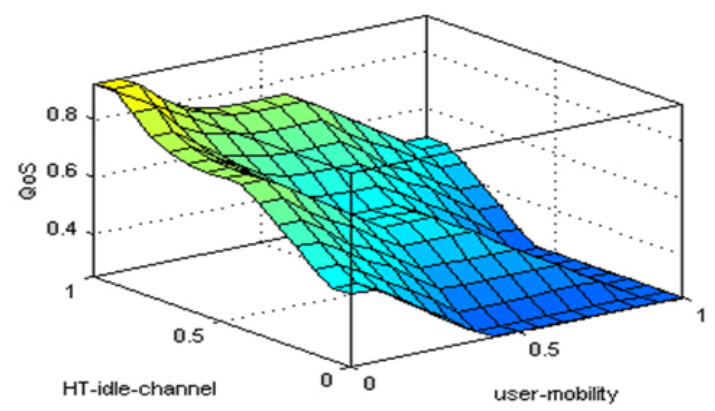

Fig. 8 : Impact analysis of user-mobility and HT-idle-channel over QoS

\section{Conclusion}

The rule-based fuzzy algorithm presented in this paper analyzed the impact of some parameters over QoS of communicating cognitive users. The presented algorithm is being analyzed in fuzzy toolbox of Matlab. The results reveal the dependence of these parameters over QoS in cognitive radio networks.

\section{Acknowledgement}

I would like to acknowledge and express my heartiest gratitude to my Ph. D supervisor Professor MoinUddin who introduced me to the very interesting subject "Cognitive Radio Technology". I have benefitted tremendously from his vision, technical insight and valuable suggestions. Prof. Moin Uddin is a senior member, IEEE. He has more than 35 years 
of experience in academics and research and has served at key Positions as Director, NIT Jallandhar, Pro-Vice-Chancellor, Delhi Technological University.
No funding agency has provided financial support for this research work.

\section{References}

1. FCC, Notice of proposed rulemaking and order, No. 03-222, Dec. 2003.

2. Mitola J. Cognitive Radio: An Integrated Agent Architecture for Software Defined Radio. Ph.D. Dissertation: KTH Royal Institute of Technology; 2000.

3. Arslan H. Cognitive radio, software defined radio, and adaptive wireless systems. Springer, 2007(e-book).

4. Liao Y., Wang T., Song L., Han Z. Listenand-Talk: Protocol Design and Analysis for Full-duplex Cognitive Radio Networks. IEEE Trans. on Vehicular Technology, Jan. 2017; 66(1): 656-67.

5. Sharma S. K., Bogale T. E., Le L. B., Chatzinotas S., Wang X., Ottersten B. TwoPhase Concurrent Sensing and Transmission Scheme for Full Duplex Cognitive Radio. in Proc. IEEE VTC Spring, Sept. 2016.

6 Boulogeorgos A.-A. A., Sofotasios P. C., Selim B., Muhaidat S., Karagiannidis G. K., and Valkama M. Effects of RF impairments in communications over cascaded fading channels. IEEE Transactions on Vehicular Technology, 2016; 65(11): 1-17.

7. Li B., Sun M., Li X., Nallanathan A., and Zhao C. Energy Detection based Spectrum Sensing for Cognitive Radios over Time-Frequency Doubly Selective Fading Channels. IEEE Transactions on signal processing, Jan. 2015; 63(2): 402-17.

8. Afifi W. and Krunz M. Incorporating selfinterference suppression for full-duplex operation in opportunistic spectrum access systems. IEEE Trans. Wireless Commun., Apr. 2015;14(4): 2180-91.

9. $\quad \mathrm{Fu} X$. , Zhou W., Xu J., Song J. Extended mobility management challenges over cellular networks combined with cognitive radio by using multi-hop network. Proceedings of International Conference on Software Engineering, Artificial Intelligence, Networking, and Parallel/ distributed Computing, July 2007; 2:683-8.

10. Liu H. J., Wang Z.X., Li S.F., Yi M. Study on the performance of spectrum mobility in cognitive wireless network. Proceedings of $11^{\text {th }}$ IEEE International Conference on Communication Systems (ICCS), 2008: 1010-4.

11. ITU-T Recommendations. Terms and definitions related to Quality of Service and network performance including dependability. ITU-T Recommendation E.800, August 1994.

12. ETSI. Network aspects (NA): general aspects of Quality of Service and network performance. ETSI Technical Report, ETR 003, 2 ${ }^{\text {nd }}$ Edition, October 1994.

13. ETSI. Satellite earth stations and systems, broadband satellite multimedia IP. IP Internetworking over Satellite: Performance, Availability and Quality of Service, March 2003, ETSI Technical Report.

14. Hardy W.C. QoS measurement and evaluation of telecommunications Quality of Service. John Wiley and Sons, England, 2001.

15. Dahi S, Tabbane S. Radio resource management on the basis of temporal characterization of spectrum holes in cognitive radio networks. Proceedings of $14^{\text {th }}$ International Symposium on Wireless Personal Multimedia Communication (WPMC), 2011: 1-5.

16. Le H-S. T. and Liang Q. An Efficient Power Control Scheme for Cognitive Radios. Proceedings of Wireless Communications \& Networks Conference (WCNC), 2007; 255963.

17. Baldo N. and Zorzi M. Fuzzy Logic for Cross Layer Optimization in Cognitive Radio Networks. EEE Communication Magazine,2008; 64-72.

18. Le H-S T. and Ly H. D. Opportunistic Spectrum Access using Fuzzy Logic for Cognitive Radio Networks. $2^{\text {nd }}$ International Conference on Communications and Electronics 
(ICCE),2008; 240-5.

19. Kaur P., Moin Uddin and Khosla A. Fuzzy Based Adaptive Bandwidth Allocation Scheme in Cognitive Radio Networks. International Conference on ICT and Knowledge Engineering, 2010; 41-5.

20. Giupponi L. and Perez-Neira A.I. Fuzzy Based Spectrum Handoff in Cognitive Radio Networks. $3^{\text {rd }}$ International Conference. on Cognitive Radio Oriented Wireless Networks and Communications CrownCom, 2008: $1-6$.

21. Lala Nisar A., Moin Uddin and Sheikh N. A. Novel Spectrum Handoffin Cognitive Radio Networks Using Fuzzy Logic. International Journal of Information Technology and computer Science, 2013; 5(11): 103-10.

22. Wanbin T. and Dong P. Spectrum Handoff in Cognitive Radio with Fuzzy Logic Control. Journal of Electronics (China), 2010; 70814.

23. Lala Nisar A., Moin Uddin and SheikhN. A. Identification and Integration of QoS parameters in Cognitive Radio Networks using Fuzzy Logic. International Journal of Emerging Sciences,2013; 3(3): 279-88.

24. Lala Nisar A., Moin Uddin and Sheikh N. A. A Novel Algorithm for Estimation of QoS in Cognitive Radio Using Fuzzy Logic.International Journal of Information Technology and Electrical Engineering,2013; 2(5):1-5.

25. Lala Nisar A., Balkhi Altaf A., Mir G.M. and Simnani R.A. Quality of Service Provisioning in Cognitive Radio Network. Oriental Journal of Computer Science \& Technology, 2017; 10(4): 780-7.

26. Hong D. and RappaportS. S. Traffic model and performance analysis for cellular mobile radio telephone systemswith prioritized and non-prioritized handoff procedure. IEEE Transactions on Vehicular Technology, 1986; VT-35(3): 448-61.

27. Stojmenovic I. Handoff of Wireless Networks and Mobile Computing. Wiley India Edition, 2002. 